\title{
A new survey for high velocity HI detections in the Southern Hemisphere
}

\author{
R. Morras ${ }^{\star, \star \star}$, E. Bajaja ${ }^{\star \star}$, E.M. Arnal ${ }^{\star \star, \star \star \star}$, and W.G.L. Pöppel ${ }^{\star \star}$ \\ Instituto Argentino de Radioastronomía, c.c. 5, (1894) Villa Elisa, Argentina \\ e-mail: rimorras@isis.unlp.edu.ar; bajaja@orbis.conae.gov.ar; arnal@iar.unlp.edu.ar; wpoppel@iar.unlp.edu.ar
}

Received July 27; accepted October 22, 1999

\begin{abstract}
A new high-sensitivity HI survey of the southern sky was made south of Declination $-25^{\circ}$, at the Instituto Argentino de Radioastronomía (IAR), on a halfdegree grid in galactic coordinates. A total of 50980 positions were observed. One of the goals of this survey was to search for high velocity clouds (HVCs). The HI profiles have been smoothed to a velocity resolution of $8 \mathrm{~km} \mathrm{~s}^{-1}$. The resulting rms noise falls in the range 0.015 to $0.020 \mathrm{~K}$. We have detected 6848 high velocity (HV) components.
\end{abstract}

Key words: surveys — ISM: clouds - ISM: kinematics and dynamics - Galaxy: halo — Galaxy: kinematics and dynamics

\section{Introduction}

The origin of the neutral-hydrogen HVCs has been an enigma for the last 36 years. The main problem in the study of these objects are the distances: they are unknown for most of the HVCs and, therefore, few of the basic physical properties of the clouds can be determined. For a review of problems and progress, see Wakker \& van Woerden (1997).

The northern sky has been surveyed several times looking for HVCs (see Table 1 in the review of Wakker \& van Woerden 1997). Recently, Hartmann \& Burton (1997) finished the Leiden-Dwingeloo HI survey made with a finer grid and higher velocity resolution than previous ones. This survey can be used for the creation of a catalogue for all the anomalous-velocity gas. However, for the southern sky the investigation of HVCs was restricted to the

Send offprint requests to : R. Morras

* Member of the Carrera del Investigador Científico of the Consejo National de Investigaciones Científicas y Técnicas of Argentina.

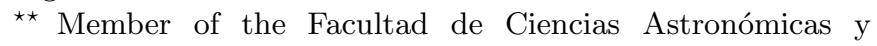
Geofísicas of the Universidad Nacional de La Plata. use of old surveys. It was first surveyed for HVCs by Mathewson et al. 1974), using the 18-m reflector at the Parkes Observatory of CSIRO (HPBW $\sim 48^{\prime}$ ), every 2.5 to 5 degrees in declination and moderate sensitivity (typically $0.3 \mathrm{~K}$ ). The main result of this survey was the finding of the extended HI complex known as the Magellanic Stream. Later, Bajaja et al. (1985) made a new survey using the 30 -m IAR dish (HPBW $\sim 34^{\prime}$ ), on a $2^{\circ} \times 2^{\circ}$ grid and a detection limit of $0.08-0.10 \mathrm{~K}$. As a result of these observations, a large number of small clouds was discovered. As we can see, a drawback of these surveys was the fact that the antenna beams were smaller than the observed grid spacing. More recently, Putman \& Gibson (1999a, 1999b) presented the first results from their complete, fully sampled, southern sky survey for HVCs, as part of the HI Parkes All-Sky Survey $\left(\delta \leqq 0^{\circ}\right)$. As they mentioned, their initial results are revealing intricate details in the spatial structure of southern HVCs, a large population of compact HVCs, and filamentary connections between previously assumed discrete clumps. Thus, until these data become of public domain, the data available at present on HVCs are quite insufficient for statistical purposes.

We decided to use the new general HI survey from the southern hemisphere, recently completed by Arnal et al. (2000), to make a more complete and homogeneous new survey of HVCs which will provide reliable and unbiased statistical information on these enigmatic objects.

\section{The data}

The new general HI survey of the southern sky was performed, using the $30-\mathrm{m}$ IAR dish (HPBW $\sim 30^{\prime}$ ), at Dec $\leq-25^{\circ}$ on a $0.5 \times 0.5$ grid in galactic coordinates. The spectrometer velocity coverage was $1055 \mathrm{~km} \mathrm{~s}^{-1}$ centered at $V_{\mathrm{LSR}}=0 \mathrm{~km} \mathrm{~s}^{-1}$, a velocity resolution of $1.27 \mathrm{~km} \mathrm{~s}^{-1}$, with channels separated $1.05 \mathrm{~km} \mathrm{~s}^{-1}$, and a rms noise $\leqq 0.07 \mathrm{~K}$ (Arnal et al. 2000). For the final 


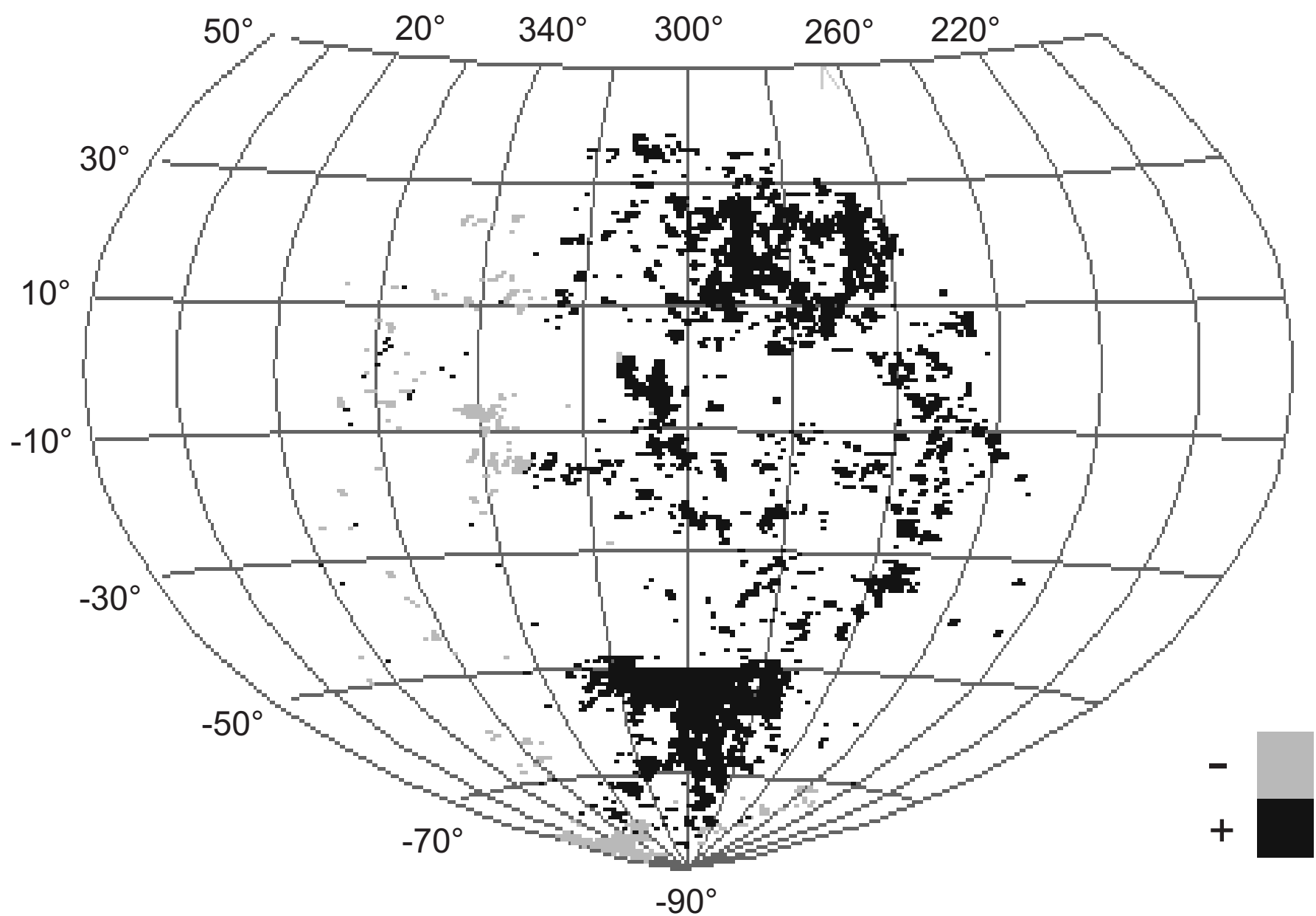

Fig. 1. Sky distribution of the $6848 \mathrm{HV}$ components detected from the IAR HI survey with velocities $V_{\mathrm{LSR}} \geq 80 \mathrm{~km} \mathrm{~s}^{-1}$. Black and grey dots are for positive and negative velocities, respectively

survey Arnal et al. adopted a velocity range of -450 to $400 \mathrm{~km} \mathrm{~s}^{-1}$, with channels spaced $1.03 \mathrm{~km} \mathrm{~s}^{-1}$, in order to fit the parameters of the northern HI survey (Hartmann \& Burton 1997). For the search of HV detections, however, we used the original survey with an effective velocity range coverage of $900 \mathrm{~km} \mathrm{~s}^{-1}$, between -450 and $450 \mathrm{~km} \mathrm{~s}^{-1}$.

The individual profiles of the IAR survey were smoothed to a velocity resolution of $8 \mathrm{~km} \mathrm{~s}^{-1}$ in order to have a better sensitivity ( $\mathrm{rms} \sim 0.015-0.020 \mathrm{~K}$ ). This sensitivity is comparable to that of the earlier HVC surveys (Bajaja et al. 1985; Hulsbosch \& Wakker 1988), but with a finer grid and better velocity resolution. For the search, the smoothed profiles were scanned visually to detect the presence of HI components at HV. We selected all the HV components whose brightness temperatures were greater than $0.08 \mathrm{~K}$ and whose absolute radial velocity in the LSR system, $V_{\mathrm{LSR}}$, were greater than $80 \mathrm{~km} \mathrm{~s}^{-1}$. About 200 of the weakest isolated components, were reobserved in order to confirm them.

During the search of the HV material we found many HV components at low galactic latitudes. However, when we look for them in the $b$ vs. $V_{\mathrm{LSR}}$ diagrams, in the latitude
Table 1. Identified galaxies

\begin{tabular}{crcl}
\hline$l\left(^{\circ}\right)$ & $b\left(^{\circ}\right)$ & $V_{\mathrm{LSR}}$ & Name \\
\hline 262.10 & 23.07 & 441 & NGC 3109 \\
273.08 & -70.29 & 364 & ESO 245-5 \\
273.28 & 13.78 & 270 & LEDA0100728 \\
299.20 & -79.42 & 178 & NGC 300 \\
328.55 & 17.85 & 320 & IC 4662 \\
332.67 & -76.74 & 119 & NGC 55 \\
343.92 & -50.19 & 121 & IC 5152 \\
\hline
\end{tabular}

range $-10^{\circ} \leq b \leq 10^{\circ}$, we found that most of them were directly connected with features belonging to the galactic spiral structure at the same velocity range. They were eliminated from the final database. Gas identified as belonging to the southern galaxies listed in Table 1 was also not included in the database. These galaxies were obtained from Paturel (1996). The galaxies quoted by Côté et al. (1997) and Mateo (1998) were also checked for any matching with our detections. 


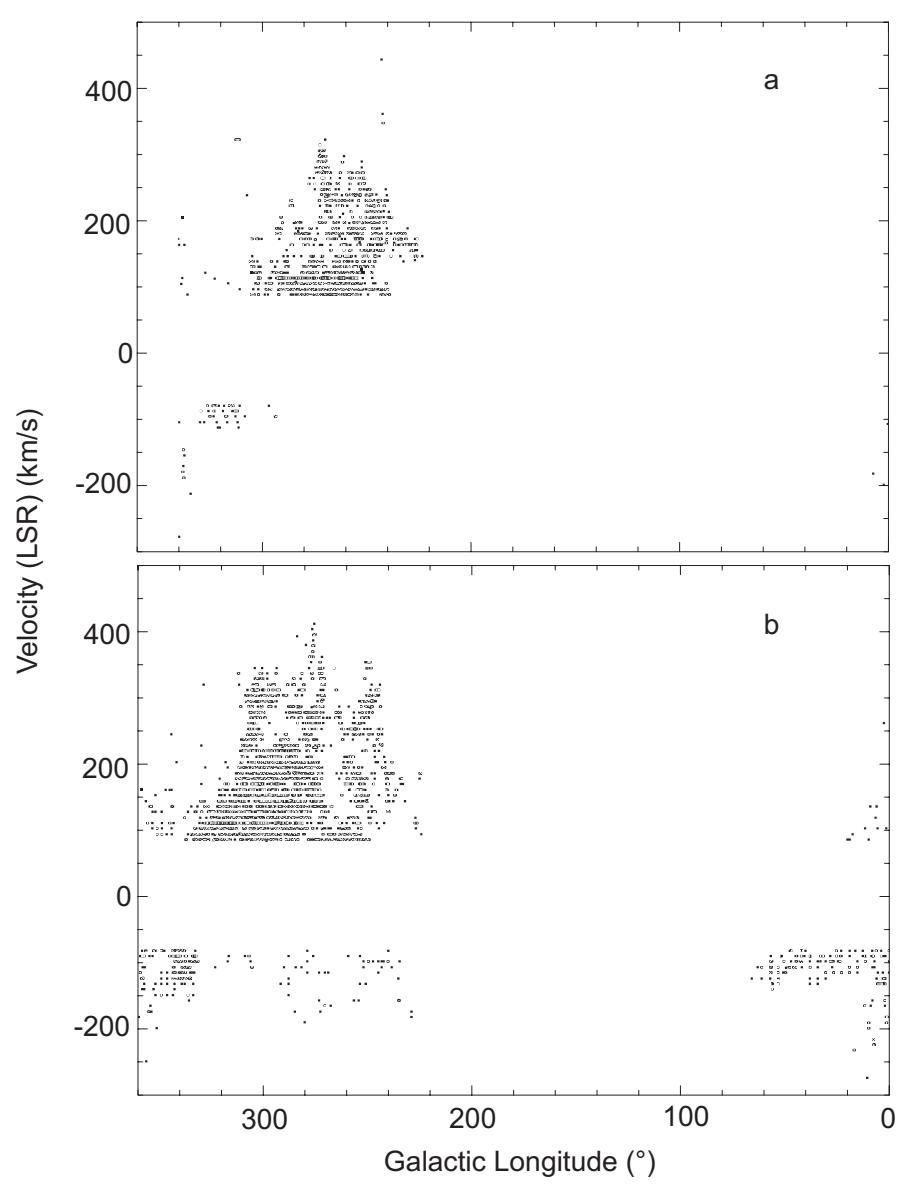

Fig. 2. LSR velocity-longitude diagrams from the IAR data for a) the northern and b) southern hemisphere

\section{Results}

The parameters of the $6848 \mathrm{HV}$ components, which were considered as reliable within $4 \mathrm{rms}$ noise, are given in Table 2. Due to the editorial policy of Astronomy and Astrophysics we publish this table, which comprises 61 printed pages, in electronic form ${ }^{1}$. Thus, only a sample page (The first page of Table 2) is given here. The first column of this table gives the HV detection number; the galactic coordinates are given in the second and third columns; the velocities in the Local (LSR) and Galactic (GSR) standard of rest systems are given in the fourth and fifth columns (the latter velocity is defined as $\left.V_{\mathrm{GSR}}=V_{\mathrm{LSR}}+220 \sin l \cos b\right)$. The sixth column gives the brightness temperature of the peak. Figure 1 shows the distribution of these $\mathrm{HV}$ components, on an Aitoff projection of galactic coordinates, making a distinction between the 6304 positive (black) and the 544 negative (grey) velocities. The cut off observed in the

\footnotetext{
1 Table 2 (full version) is only available in electronic form at the CDS via anonymous ftp to cdsarc.u-strasbg.fr (130.79.128.5) or via http://cdsweb.ustrasbg.fr/Abstract.html
}

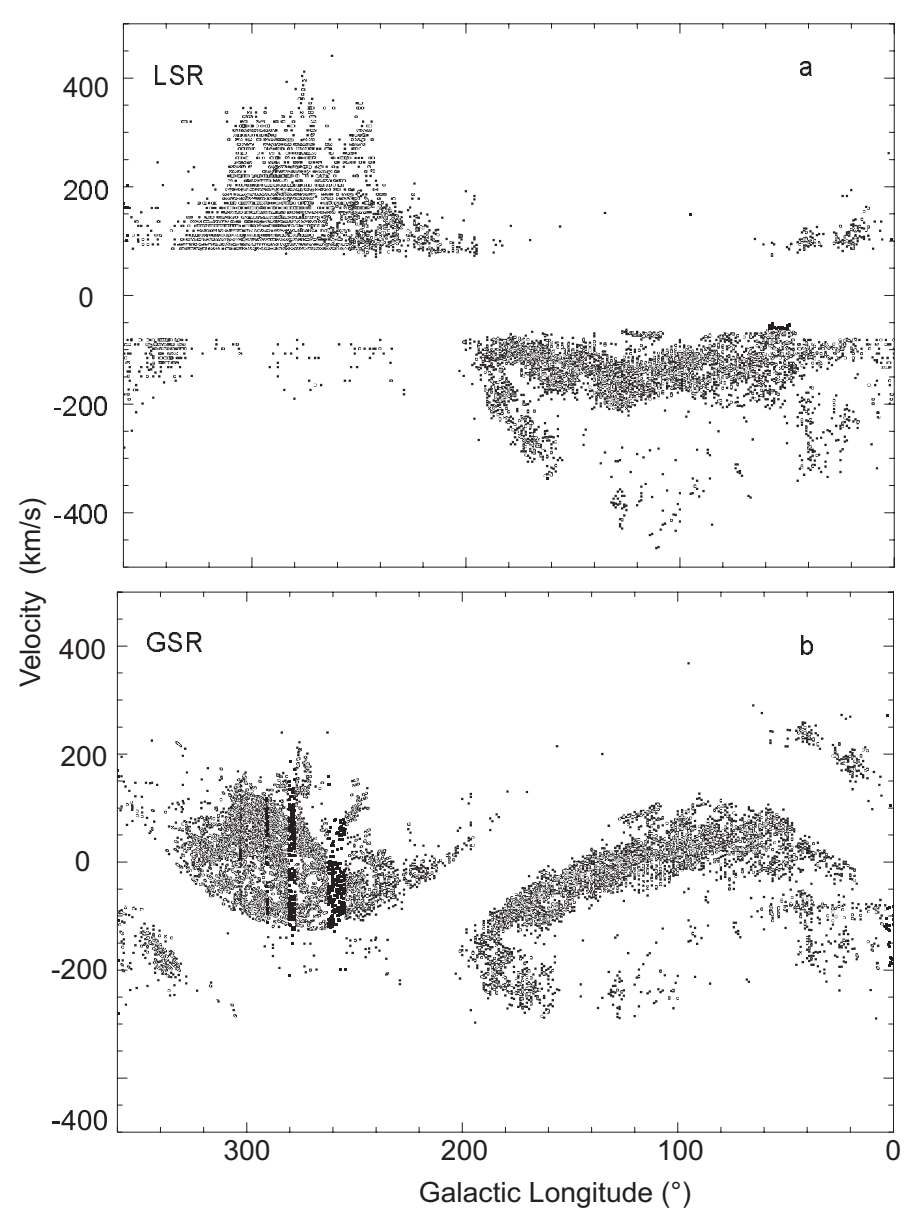

Fig. 3. a) LSR and b) GSR velocity-longitude diagrams from the IAR and Hulsbosch \& Wakker's (1988) data

figure, at latitude $-50^{\circ}$, in the region of the Magellanic Clouds, the Bridge and their inmediate surroundings, appears there because only those HV components not detected by Mathewson et al. (1974) in these structures have been included in Table 2. As is known from the work of Wannier et al. (1972) and Mathewson et al. (1974), positive velocities are prevalent in the southern sky and most of the HV components are concentrated in large clouds (Giovanelli \& Haynes 1976; Cohen \& Ruellas Mayorga 1980; Morras 1982; Morras \& Bajaja 1983; Bajaja et al. 1989; Cavarischia \& Morras 1989; etc.). However, as can be seen in the figure, many $\mathrm{HV}$ components with negative velocities are also present in the southern sky. Most of the new detections are associated with previously known HVC complexes, except for a few which seem to be compact and apparently isolated HVCs.

One of the most remarkable features observed in the figure seems to be the ring-like structure, centered at $l \simeq 280^{\circ} ; b \simeq 20^{\circ}$ (Morras et al. 1999). It includes both of the most prominent and known HVCs with positive velocities in the region, i.e., HVC $267.5+21+222$ (Cavarischia \& Morras 1989) and HVC 287.5+22.5+240 (Morras \& 
Table 2. The catalogue

\begin{tabular}{|c|c|c|c|c|c|c|c|c|c|c|c|}
\hline Number & $l\left(^{\circ}\right)$ & $b\left(^{\circ}\right)$ & $V_{\mathrm{LSR}}$ & $V_{\mathrm{GSR}}$ & $T_{b}$ & Number & $l\left(^{\circ}\right)$ & $b\left(^{\circ}\right)$ & $V_{\mathrm{LSR}}$ & $V_{\mathrm{GSR}}$ & $T_{b}$ \\
\hline 1 & 0.0 & -86.5 & -82 & -82 & 0.65 & 56 & 37.6 & -83.5 & -90 & -74 & 1.49 \\
\hline 2 & 8.1 & -86.5 & -82 & -80 & 0.17 & 57 & 42.3 & -83.5 & -98 & -81 & 1.29 \\
\hline 3 & 16.2 & -86.5 & -82 & -78 & 0.16 & 58 & 47.0 & -83.5 & -98 & -79 & 0.95 \\
\hline 4 & 48.6 & -86.5 & -90 & -81 & 0.13 & 59 & 51.7 & -83.5 & -90 & -70 & 0.25 \\
\hline 5 & 56.7 & -86.5 & -90 & -78 & 0.12 & 60 & 56.4 & -83.5 & -115 & -94 & 0.55 \\
\hline 6 & 6.3 & -86.0 & -82 & -80 & 0.68 & 61 & 61.1 & -83.5 & -124 & -102 & 0.63 \\
\hline 7 & 12.6 & -86.0 & -90 & -86 & 0.71 & 62 & 65.8 & -83.5 & -124 & -101 & 0.14 \\
\hline 8 & 18.9 & -86.0 & -90 & -85 & 0.46 & 63 & 7.6 & -83.0 & -90 & -86 & 0.61 \\
\hline 9 & 25.2 & -86.0 & -90 & -83 & 0.41 & 64 & 11.4 & -83.0 & -98 & -92 & 0.62 \\
\hline 10 & 31.5 & -86.0 & -90 & -81 & 0.25 & 65 & 15.2 & -83.0 & -98 & -90 & 0.94 \\
\hline 11 & 37.8 & -86.0 & -90 & -80 & 0.29 & 66 & 19.0 & -83.0 & -107 & -98 & 1.12 \\
\hline 12 & 44.1 & -86.0 & -90 & -79 & 0.15 & 67 & 22.8 & -83.0 & -90 & -79 & 0.81 \\
\hline 13 & 56.7 & -86.0 & -98 & -85 & 0.18 & 68 & 26.6 & -83.0 & -98 & -85 & 1.17 \\
\hline 14 & 12.6 & -85.5 & -82 & -78 & 0.35 & 69 & 30.4 & -83.0 & -98 & -84 & 0.73 \\
\hline 15 & 18.9 & -85.5 & -82 & -76 & 0.46 & 70 & 34.2 & -83.0 & -98 & -82 & 1.09 \\
\hline 16 & 25.2 & -85.5 & -82 & -74 & 0.36 & 71 & 38.0 & -83.0 & -98 & -81 & 1.44 \\
\hline 17 & 31.5 & -85.5 & -90 & -80 & 0.21 & 72 & 41.8 & -83.0 & -98 & -80 & 1.86 \\
\hline 18 & 37.8 & -85.5 & -90 & -79 & 0.21 & 73 & 45.6 & -83.0 & -107 & -87 & 1.56 \\
\hline 19 & 44.1 & -85.5 & -82 & -70 & 0.18 & 74 & 49.4 & -83.0 & -107 & -86 & 1.51 \\
\hline 20 & 50.4 & -85.5 & -98 & -84 & 0.11 & 75 & 53.2 & -83.0 & -107 & -85 & 0.59 \\
\hline 21 & 56.7 & -85.5 & -98 & -83 & 0.16 & 76 & 57.0 & -83.0 & -115 & -92 & 1.26 \\
\hline 22 & 63.0 & -85.5 & -107 & -91 & 0.14 & 77 & 287.8 & -83.0 & -149 & -174 & 0.16 \\
\hline 23 & 25.2 & -85.0 & -90 & -81 & 0.30 & 78 & 287.8 & -83.0 & 94 & 68 & 0.16 \\
\hline 24 & 37.7 & -85.0 & -107 & -95 & 0.11 & 79 & 291.6 & -83.0 & -132 & -156 & 0.12 \\
\hline 25 & 44.1 & -85.0 & -98 & -84 & 0.17 & 80 & 291.6 & -83.0 & 103 & 78 & 0.35 \\
\hline 26 & 50.4 & -85.0 & -98 & -83 & 0.12 & 81 & 348.6 & -83.0 & -82 & -87 & 1.05 \\
\hline 27 & 56.7 & -85.0 & -107 & -90 & 0.20 & 82 & 352.4 & -83.0 & -82 & -85 & 0.54 \\
\hline 28 & 303.3 & -85.0 & 102 & 85 & 0.15 & 83 & 356.2 & -83.0 & -82 & -83 & 0.54 \\
\hline 29 & 23.5 & -84.5 & -90 & -81 & 0.45 & 84 & 0.0 & -82.5 & -82 & -82 & 0.57 \\
\hline 30 & 28.2 & -84.5 & -90 & -80 & 0.99 & 85 & 3.8 & -82.5 & -82 & -80 & 0.60 \\
\hline 31 & 32.9 & -84.5 & -90 & -78 & 0.62 & 86 & 7.6 & -82.5 & -90 & -86 & 0.50 \\
\hline 32 & 47.0 & -84.5 & -82 & -66 & 0.21 & 87 & 11.4 & -82.5 & -98 & -92 & 0.57 \\
\hline 33 & 51.7 & -84.5 & -90 & -73 & 0.13 & 88 & 15.2 & -82.5 & -107 & -99 & 0.92 \\
\hline 34 & 56.4 & -84.5 & -107 & -89 & 0.18 & 89 & 19.0 & -82.5 & -107 & -97 & 1.07 \\
\hline 35 & 61.1 & -84.5 & -107 & -88 & 0.17 & 90 & 22.8 & -82.5 & -115 & -103 & 0.69 \\
\hline 36 & 280.1 & -84.5 & -190 & -210 & 0.11 & 91 & 26.6 & -82.5 & -115 & -102 & 1.31 \\
\hline 37 & 303.6 & -84.5 & 111 & 93 & 0.15 & 92 & 30.4 & -82.5 & -115 & -100 & 1.53 \\
\hline 38 & 308.3 & -84.5 & 111 & 94 & 0.34 & 93 & 34.2 & -82.5 & -107 & -90 & 1.52 \\
\hline 39 & 313.0 & -84.5 & 103 & 87 & 0.15 & 94 & 38.0 & -82.5 & -107 & -89 & 1.02 \\
\hline 40 & 23.5 & -84.0 & -98 & -88 & 1.15 & 95 & 41.8 & -82.5 & -107 & -87 & 1.95 \\
\hline 41 & 28.2 & -84.0 & -98 & -87 & 1.70 & 96 & 45.6 & -82.5 & -107 & -86 & 1.73 \\
\hline 42 & 32.9 & -84.0 & -90 & -77 & 1.71 & 97 & 49.4 & -82.5 & -107 & -85 & 1.58 \\
\hline 43 & 37.6 & -84.0 & -90 & -75 & 1.00 & 98 & 53.2 & -82.5 & -107 & -84 & 0.91 \\
\hline 44 & 42.3 & -84.0 & -98 & -82 & 0.41 & 99 & 57.0 & -82.5 & -107 & -82 & 1.39 \\
\hline 45 & 47.0 & -84.0 & -82 & -65 & 0.30 & 100 & 287.8 & -82.5 & -132 & -159 & 0.12 \\
\hline 46 & 51.7 & -84.0 & -90 & -71 & 0.10 & 101 & 287.8 & -82.5 & 94 & 66 & 0.18 \\
\hline 47 & 56.4 & -84.0 & -107 & -87 & 0.26 & 102 & 291.6 & -82.5 & 94 & 67 & 0.23 \\
\hline 48 & 61.1 & -84.0 & -107 & -86 & 0.17 & 103 & 348.6 & -82.5 & -82 & -87 & 1.77 \\
\hline 49 & 284.8 & -84.0 & -174 & -196 & 0.34 & 104 & 352.4 & -82.5 & -82 & -85 & 1.31 \\
\hline 50 & 303.6 & -84.0 & 111 & 91 & 0.24 & 105 & 356.2 & -82.5 & -82 & -83 & 0.98 \\
\hline 51 & 308.3 & -84.0 & 111 & 92 & 0.35 & 106 & 0.0 & -82.0 & -82 & -82 & 0.89 \\
\hline 52 & 313.0 & -84.0 & 103 & 86 & 0.18 & 107 & 3.8 & -82.0 & -90 & -87 & 0.64 \\
\hline 53 & 23.5 & -83.5 & -98 & -88 & 1.33 & 108 & 7.6 & -82.0 & -90 & -85 & 0.43 \\
\hline 54 & 28.2 & -83.5 & -98 & -86 & 1.09 & 109 & 11.4 & -82.0 & -90 & -83 & 0.33 \\
\hline 55 & 32.9 & -83.5 & -98 & -84 & 1.42 & 110 & 15.2 & -82.0 & -107 & -98 & 0.49 \\
\hline
\end{tabular}




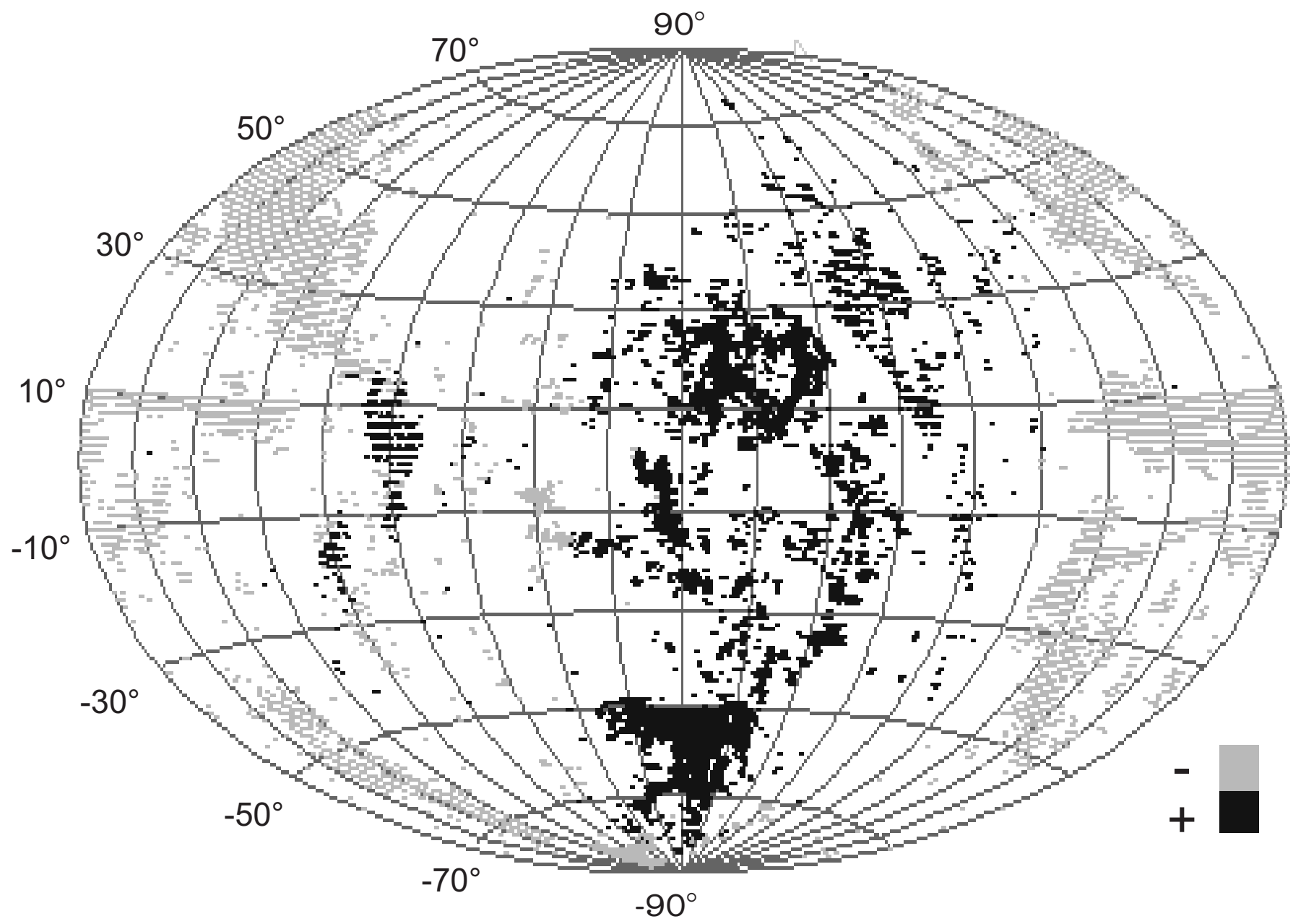

Fig. 4. Same as Fig. 1 for the whole sky from Hulsbosch \& Wakker (1988) and this database

Bajaja 1983). Part of this feature can be seen in Putman \& Gibson (1999a, 1999b).

The whole sample of HV components detected by us, including the Magellanic Stream (MS), are indicating that, at a level of $0.08 \mathrm{~K}$, the fraction of the southern sky covered by $\mathrm{HV}$ gas is about $13 \%$. This value is of the same order as the one derived by Wakker (1991), who found that at the limit of $0.05 \mathrm{~K}, 11 \%$ ( $18 \%$ when including the MS and the Outer Arm (OA)) of the sky is covered by gas having $\left|V_{\mathrm{LSR}}\right| \geq 100 \mathrm{~km} \mathrm{~s}^{-1}$. As quoted by Wakker, the MS and the OA contain a substantial part of the HV gas.

The highest positive LSR velocity found in the survey is in the detection number $2820\left(V_{\mathrm{LSR}}=412 \mathrm{~km} \mathrm{~s}^{-1}\right)$ around $l \simeq 275.5 ; b \simeq-30^{\circ}$. This detection is not isolated since there are eighteen positive detections around it in the velocity range $360 \leq V_{\mathrm{LSR}} \leq 412 \mathrm{~km} \mathrm{~s}^{-1}$ (see Table 2). This cloud is probably related to the Magellanic System. The highest negative detected velocity is $-280 \mathrm{~km} \mathrm{~s}^{-1}$ (number 4389, at $l=359.5, b=0.5$ ) which could have been originated in the activity of the galactic center.
Figures $2 \mathrm{a}$ and $\mathrm{b}$ show, in a similar way as in Giovanelli (1980), a plot of $V_{\mathrm{LSR}}$ versus galactic longitude, for the IAR data, in both galactic hemispheres. The figures show that most of the HV gas have positive velocities. They also show that the $\mathrm{HV}$ detections with negative velocities are predominantly in the southern galactic hemisphere. Comparing these figures with Fig. 1, it can be seen that a large percentage of the negative velocity gas belongs to the Magellanic Stream. The exceptions could be: 1) $z$-extensions from features connected to the galactic spiral structure which were not identified as such and, therefore, not deleted from the catalog, and 2) the HV components at low galactic longitude which were suggested as being part of the material that is falling towards the Galactic Center at distances of about $20 \mathrm{kpc}$ or more (Mirabel \& Morras 1984). In Figs. 3a and b we have plotted the distribution of the LSR and GSR velocities, respectively, as a function of the galactic longitude, of our and Hulsbosch \& Wakker's (1988) data. Figure 3a shows that the distributions of the data points are quite different in the different parts of the sky. As was already noticed by Giovanelli (1980), the $V_{\mathrm{LSR}}$ diagram of HVCs visible from 
the northern sky (which correspond to the galactic regions at $l \leq 210^{\circ}$ ) suggests the presence of two distint populations of HVCs, namely, one with relatively low velocities $\left(-150 \leq V_{\mathrm{LSR}} \leq-80 \mathrm{~km} \mathrm{~s}^{-1}\right)$ and the other one with relatively high negative values. In spite of the larger number of points available in the IAR data, no similar separation into two populations is apparent in the southern sky. The figure also shows that the highest negative velocities, in absolute values, are larger than the highest positive velocities and that both are much larger than the value of the rotational velocity currently assumed for the LSR in the Galaxy. The new IAR data confirm this asymmetry. The upper limit for the positive $V_{\mathrm{LSR}}$ velocities is about $+412 \mathrm{~km} \mathrm{~s}^{-1}$ while negative velocities reach values as high as $-465 \mathrm{~km} \mathrm{~s}^{-1}$ at $l \simeq 111^{\circ}, b \simeq-7^{\circ}$. Figure $3 \mathrm{~b}$ also shows that large systematic GSR velocities occur after subtraction of the galactic rotation component.

Finally, Fig. 4 shows the spatial distribution for the whole sky of both databases in the same kind of display as in Fig. 1. A small gap is visible between the southern and northern databases, because the IAR data were taken at Dec $\leq-25^{\circ}$ and Hulsbosch \& Wakker data at $\operatorname{Dec} \geq-17.2$.

Acknowledgements. This work was supported by the Argentinian National Research Council (CONICET) under project P.I.P 4253/96.

\section{References}

Arnal E.M., Bajaja E., Larrarte J.J., Morras R., Pöppel W.G.L., 2000, A\&AS (in press)
Bajaja E., Cappa de Nicolau C.E., Cersosimo J.C., Loiseau N., Martin M.C., Morras R., Olano C.A., Pöppel W.G.L., 1985, ApJS 58, 143

Bajaja E., Cappa de Nicolau C.E., Martin M.C., Morras R., Olano C.A., Pöppel W.G.L., 1989, A\&AS 78, 345

Cavarischia G.A., Morras R., 1989, A\&AS 78, 437

Cohen R.J., Ruelas Mayorga R.A., 1980, MNRAS 193, 583

Côté S., Freeman K.C., Carignan C., Quinn P.J., 1997, AJ 114, 1313

Giovanelli R., 1980, AJ 85, 1155

Giovanelli R., Haynes M., 1976, MNRAS 177, 525

Hartmann D., Burton W.B., 1997, Atlas of Galactic Neutral Hydrogen. Cambridge: Cambridge Univ. Press

Hulsbosch A.N.M., Wakker B.P., 1988, A\&AS 75, 191

Mateo M., 1998, ARA\&A 36, 435

Mathewson D.S., Cleary M.N., Murray J.D., 1974, ApJ 190, 291

Mirabel I.F., Morras R., 1984, ApJ 279, 86

Morras R., 1982, A\&A 115, 249

Morras R., Bajaja E., 1983, A\&AS 51, 131

Morras R., Bajaja E., Arnal E.M., Pöppel W.G.L., 1999 (in preparation)

Paturel G., 1996, The Lyon-Meudon Extragalactic Database (2nd. edition), Observatoire de Lyon

Putman M.E., Gibson B.K., 1999, Stromlo Workshop on High Velocity Clouds, ASP Conf. Ser. 166, Gibson B.K. and Putman M.E. (eds.), p. 276

Putman M.E., Gibson B.K., 1999, Proc. Astron. Soc. Aust. 16, 70

Wakker B.P., 1991, A\&A 250, 499

Wakker B.P., van Woerden H., 1997, ARA\&A 35, 217

Wannier P., Wrixon G.T., Wilson R.W., 1972, A\&A 18, 224 